\title{
Large-Area Structural Color Filtering Capitalizing on Nanoporous Metal- Dielectric-Metal Configuration
}

\author{
Yang Li, Wen-Jing Yue ${ }^{1 *}$, Zhen-Xiang Chen ${ }^{1}$, Bing-Qiang Cao ${ }^{2}$, Xiao-Qian Fu', Chun-Wei Zhang ${ }^{1}$ and Zhi-Ming Li ${ }^{1}$
}

\begin{abstract}
We present a highly efficient structural color filtering approach for large-area application, using a nanoporous anodic alumina (NAA) film overlaid with an aluminum (Al) layer on top of an optically thick Al substrate. The NAA film, consisting of a self-assembled nanopore array in a hexagonal lattice, is equivalent to a quasi-homogeneous medium according to effective medium theory. The proposed structure enables strong absorption at resonance owing to the Fabry-Perot resonance supported by the metal-dielectric-metal configuration and the plasmonic effect mediated by the top nanoporous Al layer. The reflection colors can be readily tuned by altering the NAA thickness that is determined by anodization time, thereby allowing the flexible creation of complicated color images on a single platform. By fabricating three samples with different NAA thicknesses in a large area of $2 \mathrm{~cm} \times 2 \mathrm{~cm}$, it is confirmed that the proposed color filtering scheme exhibits highly enhanced color purity and high reflection efficiency of up to $73 \%$, which is superior to that generated by previously reported NAA-based approaches. The presented strategy can pave the way for the efficient fabrication of large-area color filtering devices for various potential applications, including color display devices, imaging sensors, structural color printing, and photovoltaic cells.
\end{abstract}

Keywords: Structural color filtering, Fabry-Perot resonance, Plasmonic effect, Nanoporous anodic alumina, Metal-dielectric-metal configuration, Large-area, Effective medium theory

\section{Background}

Color filtering technologies resorting to subwavelength structures have played a vital role in a variety of fascinating applications, such as transmissive/reflective color filters in display devices, imaging systems, chromatic polarizers, photovoltaic cells, and photorealistic structural color printing [1-10]. Structural color filtering, which involves from traditional organic dyes/pigments-based chemical filters, successfully mitigates the drawbacks of chemical filters including significant performance degradation under long-standing ultraviolet illumination and serious environmental stress. Moreover, structural color filtering exhibits salient features of flexible spectral filtering properties and stable specifications. Various schemes for achieving structural colors, particularly those that

\footnotetext{
* Correspondence: ise_yuewj@ujn.edu.cn

${ }^{1}$ School of Information Science and Engineering, University of Jinan, Jinan 250022, China

Full list of author information is available at the end of the article
}

involve the use of multi-layer thin films [11-15], subwavelength-grating-enabled plasmonic or guidedmode resonance nanostructures [16-22], and metasurfaces [23], have been proposed. The fabrication of subwavelength-grating-based configurations and metasurfaces generally require complicated procedures, such as electron beam (e-beam) lithography and reactive ion etching, which are time-consuming and have high cost, and greatly limit their potential applications in large-area circumstances. Thus, multi-layer thin films, particularly the Fabry-Perot (FP) resonator with a dielectric cavity sandwiched by two metallic layers, are widely used as an alternative method. However, multiple fabrication steps are needed for the deposition of different cavity thickness for the purpose of simultaneously generating full colors on a single platform, which hinder their usage in practical applications.

To mitigate the aforementioned issues, nanoporous anodic alumina (NAA), which is one of the cost-effective 
porous self-assembled materials consisting of many parallel straight cylindrical nanopores perpendicular to an optically thick $\mathrm{Al}$ substrate, is regarded as the best candidate $[24,25]$. Several strategies for generating multiple structural colors on NAA films are currently used, including covering the top surface and inner sidewall of the NAA film with carbon or dielectric material, such as $\mathrm{TiO}_{2}$ [26-28], or depositing metallic layers on top of an NAA film [29-32]. A FP resonance-enabled asymmetric metal-dielectric-metal (MDM) configuration can be easily constructed by simply depositing a metallic layer on top of an NAA. At resonance, a strong suppression in reflection can be observed, corresponding to a specific reflection color. The metallic layer deposited on top of a NAA, which consists of a hexagonal lattice of pores, can simultaneously enable a strong plasmonic effect, further enhancing the absorption of the structure [32,33]. Through a simple adjustment of the geometry of NAA, such as thickness and pore diameter, the observed colors can be effectively tuned. However, the reported metal-coated NAA configurations, which use noble metals such as platinum and gold, lead to a high cost of device [29, 32]. And, the optical spectra of the reported configurations exhibit low reflection efficiencies, multiple resonances within the visible spectral band, or broad spectral bandwidths, resulting in undesired low color purity.

In this work, we demonstrate a highly efficient structural color filtering scheme for large-area applications by exploiting a simple nanoporous structure based on an NAA film overlapped with a thin aluminum (Al) layer. Vivid distinctive reflection colors can be readily tuned by simply altering the thickness of the NAA. Al is particularly applied because of its outstanding optical properties, including high reflectivity in the visible regions, low cost, and compatibility with the standard complementary metal-oxide-semiconductor fabrication process [20-22]. The individual role of each geometric parameter of the proposed structure is rigorously inspected through the finite-difference time-domain (FDTD) method. Samples with different NAA thicknesses were manufactured over a large area through a non-lithography method. The optical characteristics of the prepared samples were measured and evaluated by comparing the measured results with the simulated results.

\section{Methods/Experimental}

\section{Design of the Proposed Large-Area Color Filtering Scheme}

In this study, we aim to develop a highly efficient color filtering scheme for large-area application by capitalizing on a nanoporous MDM resonant configuration capable of supporting FP resonance in addition to the plasmonic effect. Figure 1a depicts the schematic configuration of the proposed MDM-structure-based color filtering device, where an NAA film is sandwiched between an optically thick $\mathrm{Al}$ substrate and a top thin $\mathrm{Al}$ layer. The thickness of the NAA and top Al layer are denoted as $t_{1}$ and $t_{2}$, respectively. For the fabrication of a nanoporous structure, the NAA film is a self-assembled porous structure that originated from an $\mathrm{Al}$ plate through a simple anodization process rather than through conventional approaches relying on complicated and expensive e-beam lithography. As shown in Fig. 1b, the NAA film consists of a hexagonal lattice of pores with the diameter of $\mathrm{d}$. The gap between two adjacent pores is represented by $\Lambda$. When the gap between two pores is sufficiently small compared with the wavelength of interest, the nanoporous layers, embracing top $\mathrm{Al}$ coating and NAA film, behave as quasi-homogeneous media. Therefore, we appropriately set $\Lambda$ and $d$ to 100 and $65 \mathrm{~nm}$, respectively. Effective medium theory has been commonly used for elucidating the properties of such nanoporous structures [34, 35].

For the FP-resonance-enabled asymmetrical MDM structure, the reflection is strongly suppressed at resonance, corresponding to a reflection dip, when the deconstructive interference occurs between the directly reflected light at the top air- $\mathrm{Al}$ interface and the resonantly coupled light within the NAA cavity. Different from the conventional MDM structure based on continuous layers [12-14], the proposed nanoporous structure is expected to tune the reflection colors by not only changing the thickness of the dielectric cavity but also altering the pore diameter or gap [28, 29]. More importantly, owing to the top nanoporous $\mathrm{Al}$ layer, the proposed structure is capable of enabling a strong plasmonic effect in addition to FP resonance, which can efficiently reinforce the absorption of the proposed structure. The proposed structure is carefully designed and assessed with a tool based on the FDTD method. The dispersion characteristics of the materials used for the simulations are derived from the built-in multi-coefficient model provided by the tool. For simplicity, the simulation region, which is denoted by a dotted red box in Fig. 1b, only contains a unit structure, and the periodic boundaries are applied for the $x$ and $y$ axes. A default auto non-uniform mesh refinement with mesh accuracy of 3 is set for the entire simulation region. This set-up ensures a good trade-off between accuracy and simulation time. A plane wave serves as a light source. We set the thickness of the top $\mathrm{Al}$ coating to $15 \mathrm{~nm}$ through a set of simulations to obtain a near-zero reflection dip in order to produce high-purity colors. Then, the spectral tunability upon the thickness of the NAA cavity is investigated, as plotted in Fig. 2a. As the NAA thickness $t_{2}$ varies from 110 to $180 \mathrm{~nm}$, the resonance wavelength slightly redshifts from 465 to $670 \mathrm{~nm}$, covering the entire visible spectral band. When the 

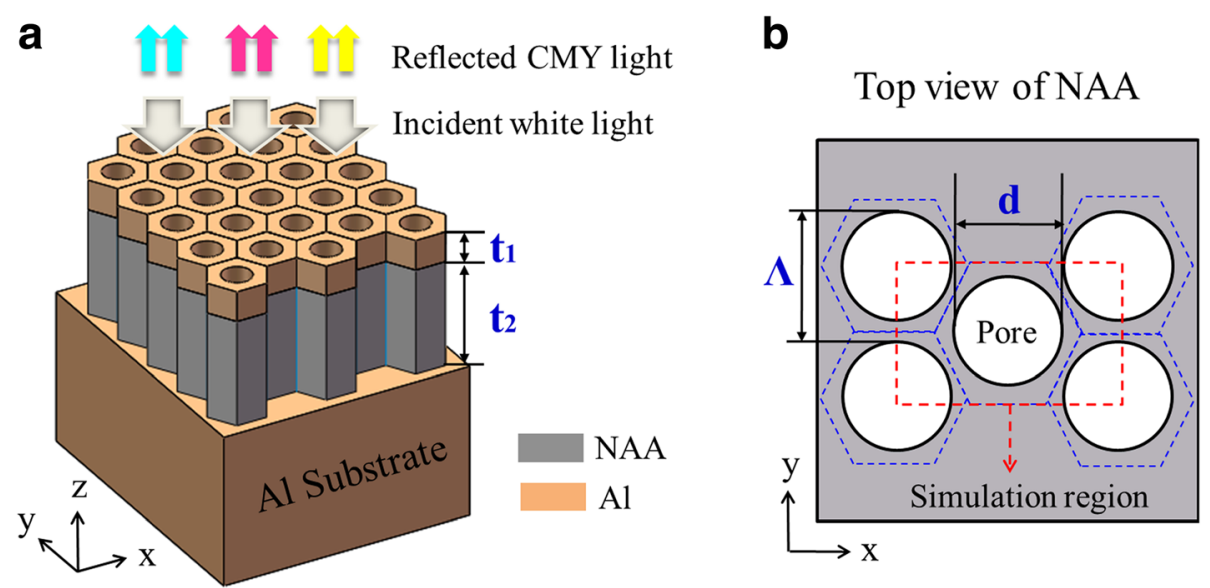

Fig. 1 a Schematic geometry of the proposed structure based on the Al-coated NAA film atop an Al substrate for large-area color filtering. $\mathbf{b}$ Top view of the NAA film with a hexagonal lattice of nanopores

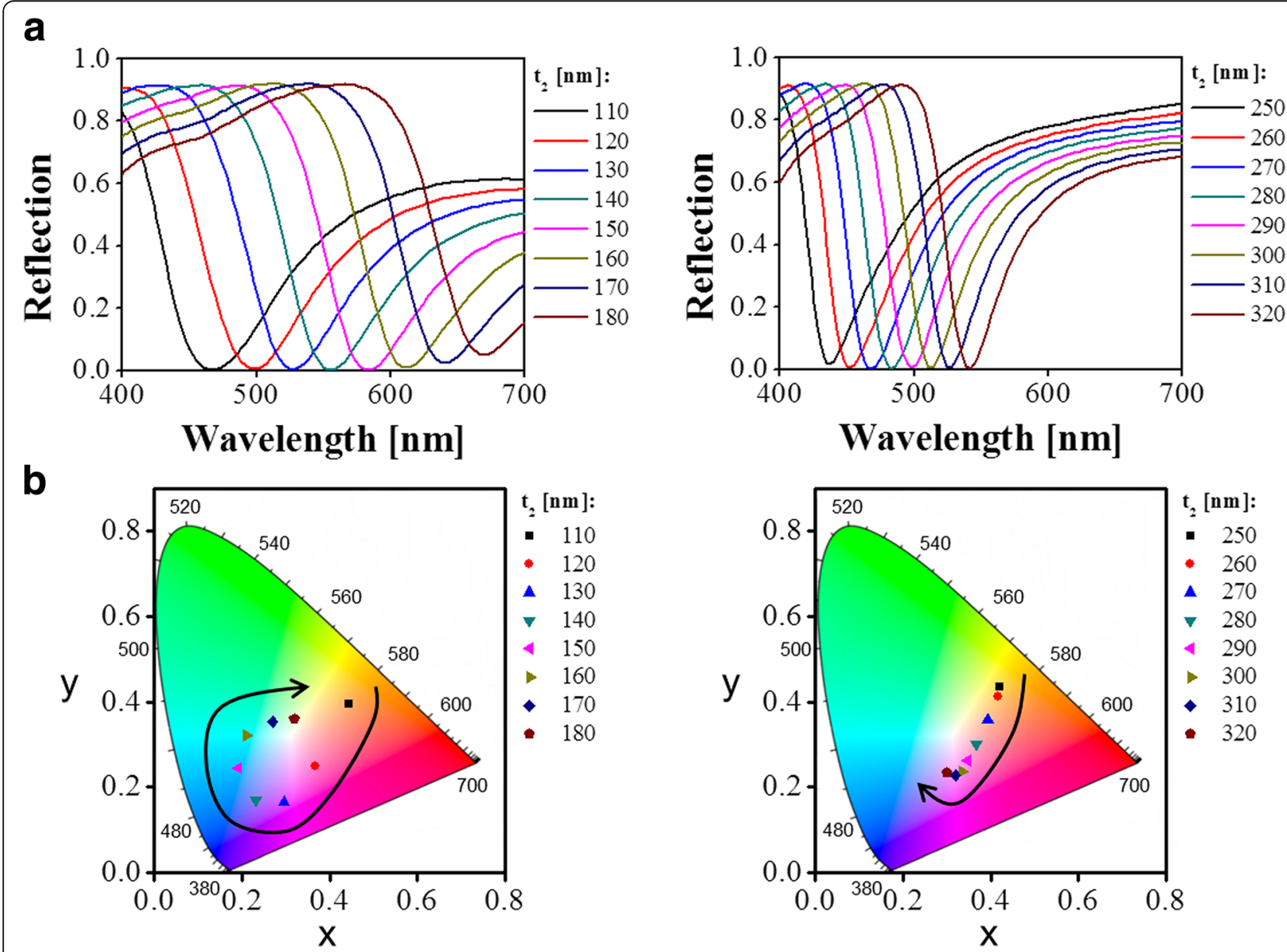

Fig. 2 a Simulated reflection spectral responses of the proposed color filtering structure with NAA thickness varying from 110 to $320 \mathrm{~nm}$. b Corresponding chromaticity coordinates in the CIE 1931 chromaticity diagram 
thickness of the NAA is further increased, the resonance dip eventually enters into the near-infrared band. Meanwhile, a higher-order resonance dip with relatively narrow bandwidth appears from the ultraviolet band to the visible band with the NAA thickness ranging from 250 to $320 \mathrm{~nm}$. It should be noted that a single resonance dip in the visible band is desired for the production of vivid reflection colors with high purity. To estimate the color purity of proposed structure, the chromaticity coordinates that correspond to the reflection spectra are calculated and mapped into the standard International Commission on Illumination (CIE) 1931 chromaticity diagram, as depicted in Fig. 2b. The chromaticity coordinates are observed to evolve along the black arrow as the thickness of the NAA increases. In particular, the circular trace of the chromaticity coordinates with NAA thickness increasing from 110 to $180 \mathrm{~nm}$ indicates that the proposed scheme is capable of achieving vivid full colors through the simple adjustment of NAA thickness. Figure 3 depicts the polarization-dependent reflection spectra of the proposed structure with different cavity thicknesses of $t_{2}=110,160$, and $320 \mathrm{~nm}$. It is observed that the same reflection spectra are maintained in terms of the resonance wavelength and reflection efficiency as the polarization angle of incident light varies from $0^{\circ}$ to $90^{\circ}$. Therefore, the proposed structure is deemed to enable the polarization-independent property, which is attributed to the symmetrical geometry of the proposed structure.

\section{Fabrication of Color Filtering Devices}

For the purpose of evaluating the proposed color filtering scheme, we manufactured three samples with different NAA thicknesses through the following fabrication processes. Commercial high-purity (99.999\%) Al foil was initially degreased in acetone and subsequently washed in isopropyl alcohol and deionized water without any other pretreatments before adonization. The prepared $\mathrm{Al}$ foil was cut into square pieces, which were placed in a self-made holder with an effective area of $2 \mathrm{~cm} \times 2 \mathrm{~cm}$ during the anodization process. The electrolyte container was a transparent beaker with a total volume $4 \mathrm{~L}$. In this experiment, a two-step anodization process was sequentially implemented. In the first step, anodization was performed by immersing the $\mathrm{Al}$ foil square pieces into $0.3 \mathrm{M}$ oxalic acid under the constant anodization voltage of $40 \mathrm{~V}$ at room temperature for $30 \mathrm{~min}$. Afterward, the anodized specimens were immersed in a mixture of $6.0 \mathrm{wt} \% \mathrm{H}_{3} \mathrm{PO}_{4}$ and $1.8 \mathrm{wt} \% \mathrm{H}_{2} \mathrm{CrO}_{4}$ at $60{ }^{\circ} \mathrm{C}$ for $5 \mathrm{~h}$ for the removal of the oxidized layers. In the second step, anodization was performed with the same experimental conditions used in the first step. As a result, the partially anodized parts of the original $\mathrm{Al}$ foil pieces were transformed into the NAA films with well-defined straight pores. An undesired optically thick alumina layer was formed within the pore at the top of the underlying $\mathrm{Al}$ foil because of the oxidation of $\mathrm{Al}$ during the second anodization step. In order to remove the undesired alumina layer within the pore completely, the anodized samples were dissolved in $6.0 \mathrm{wt} \% \mathrm{H}_{3} \mathrm{PO}_{4}$ at $60{ }^{\circ} \mathrm{C}$ for $10 \mathrm{~min}$. Finally, three samples with different NAA thicknesses of 110,160 , and $320 \mathrm{~nm}$, were prepared by accurately controlling the anodization time. The top and cross-sectional views of the fabricated NAA samples are presented in Fig. 4a, exhibiting a satisfactory nanoporous structure with well-shaped pores and high periodicity. For the prepared samples, the pore diameter and gap between two neighboring pores were measured to be $d=65 \mathrm{~nm}$ and $\Lambda=100 \mathrm{~nm}$, respectively. Then, an $\mathrm{Al}$ coating layer was deposited on top of the prepared NAA film via sputtering deposition under the base pressure of $6.7 \times 10^{-5} \mathrm{~Pa}$ and $2.0 \mathrm{~kW}$ direct current power for $260 \mathrm{~s}$. Notably, the minimum deposition rate of $0.5 \AA / \mathrm{s}$ was selected to ensure the thickness accuracy of the deposited $\mathrm{Al}$ layer. Figure 4b illustrates the top view of the scanning electron microscopy (SEM) images of
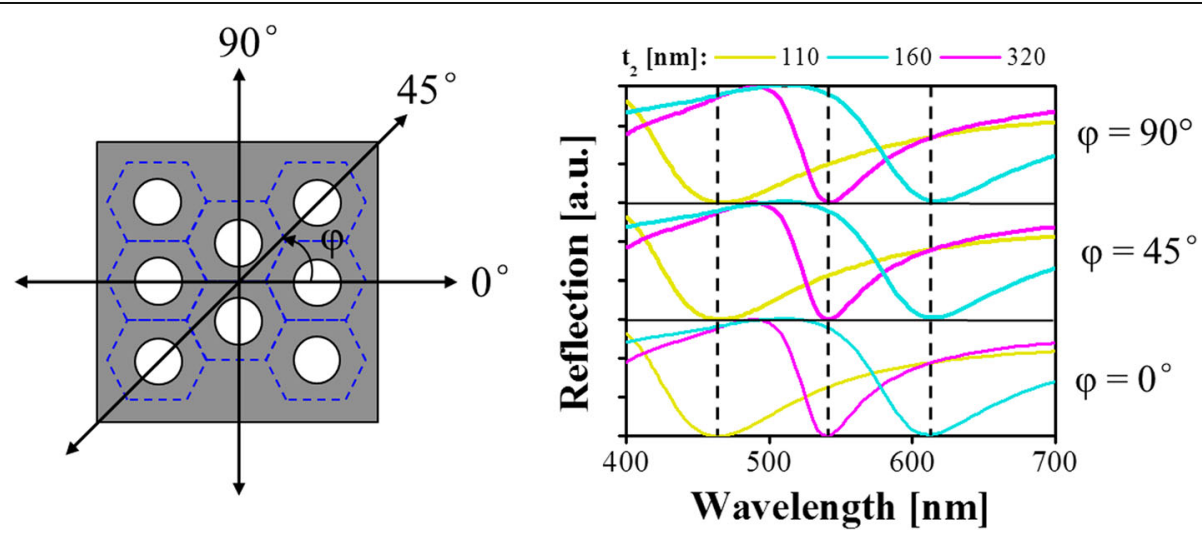

Fig. 3 Simulated reflection spectra of the proposed structure with respect to the incident polarization 


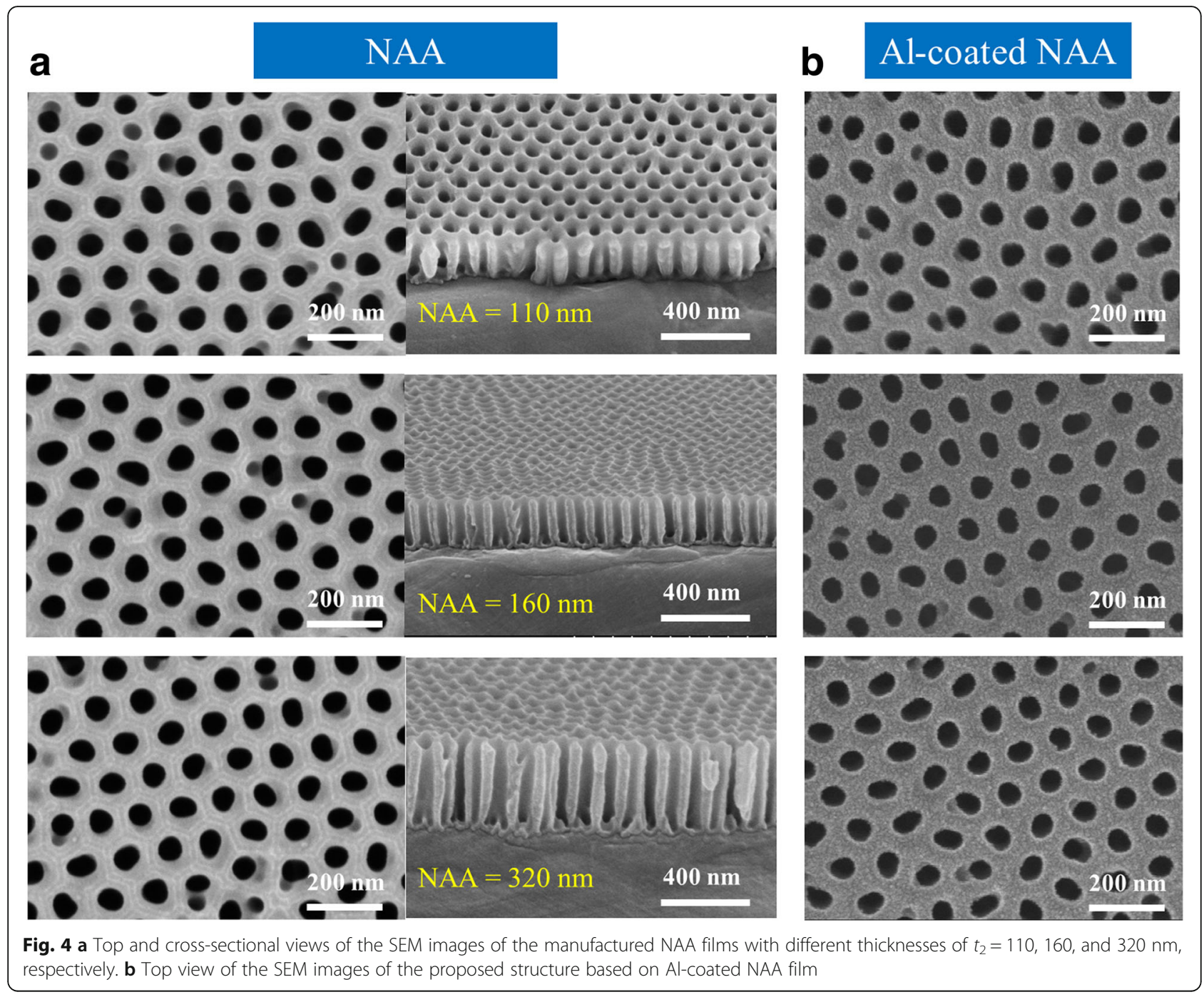

the manufactured color filtering devices with thin $\mathrm{Al}$ coating layers on top. The thickness of the deposited $\mathrm{Al}$ layers was measured to be $t_{1}=16 \mathrm{~nm}$, which is close to the designed thickness.

\section{Optical Characterization of the Prepared Color Filtering Devices}

The optical performance of each prepared sample was thoroughly assessed with respect to reflection color and spectral response. Figure 5a shows the measured reflection colors at normal incidence from the manufactured samples with large dimensions of $2 \mathrm{~cm} \times 2 \mathrm{~cm}$. Vivid primary subtractive colors of yellow, cyan, and magenta were observed, verifying that the proposed color filtering approach is capable of rendering full-color generation with highly enhanced color purity. For a better understanding of the achieved high purity, a customized experimental setup, including a halogen lamp serving as the light source, a beam splitter, and a spectrometer, was implemented to measure the reflection spectra of the prepared samples. Figure 5b, c depicts the measured reflection spectra together with the simulated reflection spectra as references, where a good correlation was observed between the experiment and the simulation with regard to resonance wavelength and shapes of the reflection spectra. A small discrepancy in spectral bandwidth and reflection efficiency can be ascribed to the imperfection of the fabrication with regard to the design, including the roughness of the Al-NAA interfaces and inconsistent periodicity and size of the pores, which could be easily observed in Fig. 4. It is also found that the fabricated samples with NAA thicknesses of 110 , 160 , and $320 \mathrm{~nm}$ had near-zero resonance dips located at wavelengths of 484,614, and $539 \mathrm{~nm}$, respectively, and practically achieved high reflection efficiencies of up to $73 \%$. The chromaticity coordinates corresponding to 


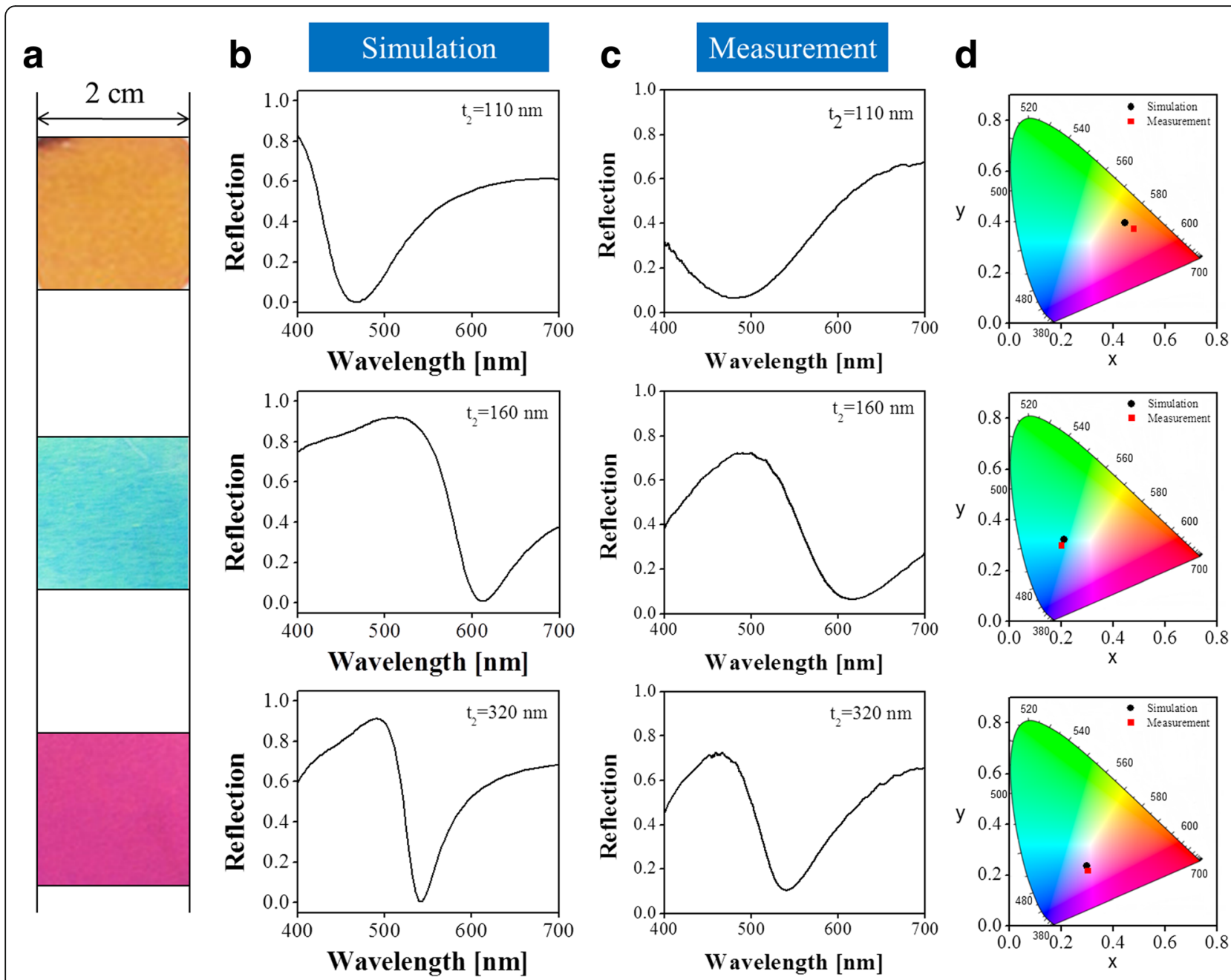

Fig. 5 a Captured optical color images at normal incidence from the manufactured devices with different NAA thicknesses of $t_{2}=110$, 160, and $320 \mathrm{~nm}$. b Simulated and (c) measured reflection spectra of the fabricated devices. d Corresponding chromaticity coordinates in the CIE 1931 chromaticity diagram in response to the simulated and measured spectra

the simulated and measured spectra were calculated and plotted in the CIE 1931 chromaticity diagram, as depicted in Fig. 5d. The observed high-purity reflection colors shown in Fig. 5a are confirmed to benefit from the achieved high reflection efficiency and near-zero reflection dip.

\section{Results and Discussion}

\section{Investigation of the Plasmonic Effect}

To examine the plasmonic effect enabled by the nanoporous $\mathrm{Al}$ layer, we thoroughly investigate the proposed structure by replacing the NAA cavity with an equivalent homogeneous cavity exhibiting an effective refractive index. Based on effective medium theory, the effective refractive index of the NAA cavity with a pore gap of $100 \mathrm{~nm}$ and a pore diameter of $65 \mathrm{~nm}$ is derived to be $n_{\text {eff }}=\sim 1.48$, according to the equation expressed as follows:

$$
\begin{aligned}
& \left(n^{2}{ }_{\text {eff }}-n^{2}{ }_{\mathrm{Al}_{2} \mathrm{O}_{3}}\right) /\left(n^{2}{ }_{\text {eff }}+2 n^{2} \mathrm{Al}_{2} \mathrm{O}_{3}\right) \\
& \quad=f_{\text {air }}\left(1-n^{2}{ }_{\mathrm{Al}_{2} \mathrm{O}_{3}}\right) /\left(1+2 n^{2} \mathrm{Al}_{2} \mathrm{O}_{3}\right)
\end{aligned}
$$

Here, the refractive index of alumina $\left(\mathrm{Al}_{2} \mathrm{O}_{3}\right)$ is $n_{\mathrm{Al} 2 \mathrm{O} 3}$ $=1.77$ and the fill fraction of air within the NAA cavity is $f_{\text {air }}=\pi(d / \Lambda)^{2} / 2 \sqrt{3}$. Figure 6a shows a comparison of the reflection spectra between the structures based on the NAA cavity and the homogeneous cavity with $n_{\text {eff }}$ of 1.48 for different cavity thicknesses of $t_{2}=110,160$, and $320 \mathrm{~nm}$. A good correlation can be observed between the two cases, indicating that the proposed structure can be safely equivalent to the structure based on a homogeneous cavity with an effective index of 1.48. For the equivalent structure based on the homogeneous cavity, the influence of pores within the top $\mathrm{Al}$ layer on the reflection spectrum is depicted in Fig. 6b. Compared with the case including no pore in the top $\mathrm{Al}$ layer, the 

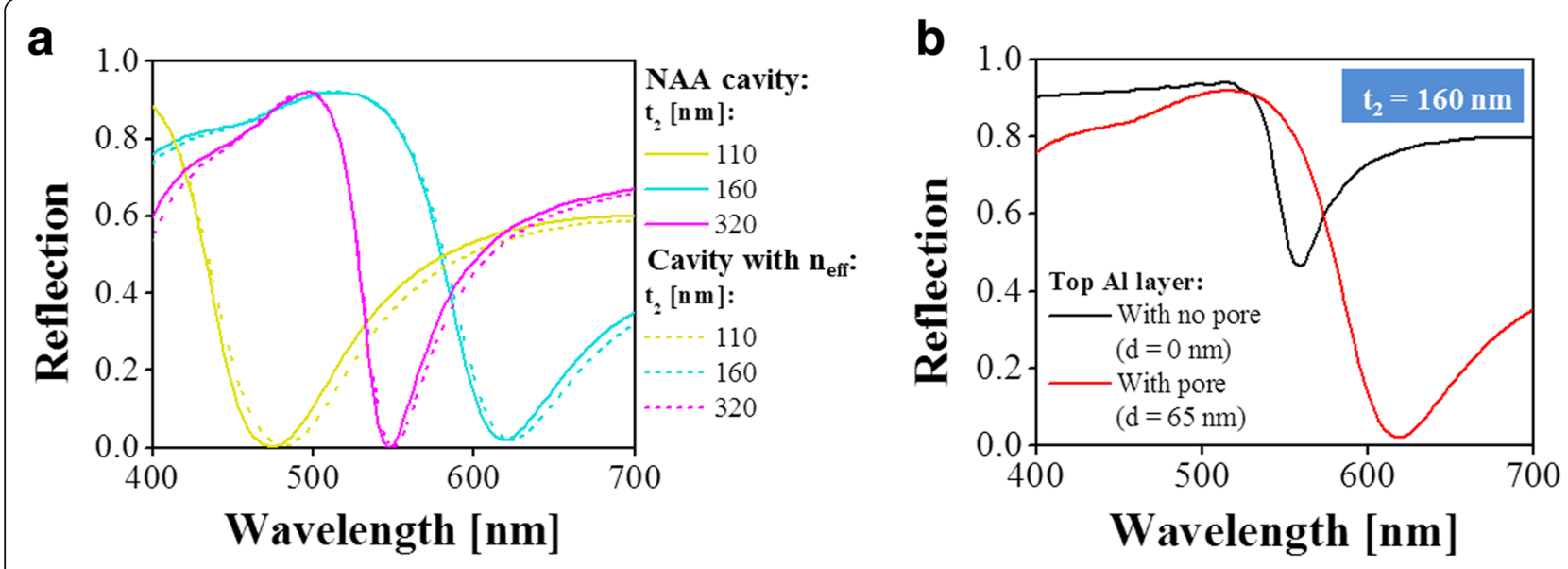

C

\section{Top Al layer with no pore}

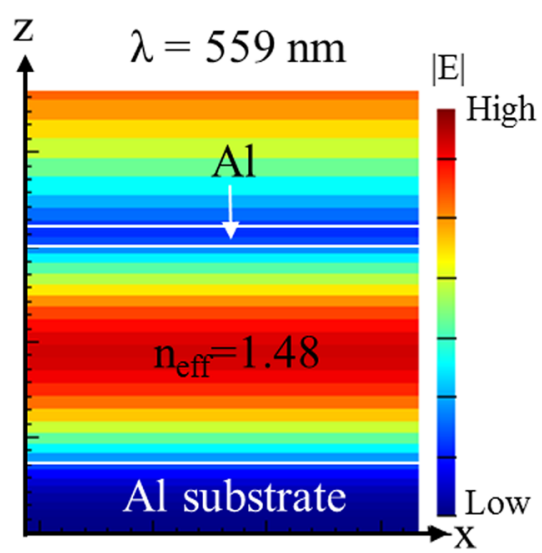

\section{Top Al layer with pore $(\mathrm{d}=65 \mathrm{~nm})$}

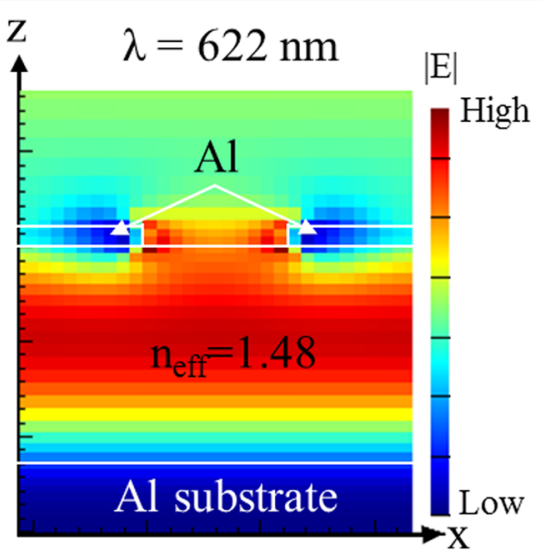

Fig. 6 a Simulated reflection spectra of the proposed structure based on the NAA cavity and the equivalent structure based on a homogeneous cavity with effective refractive index ( $n_{\text {eff }}$ ) for different cavity thicknesses of $t_{2}=110,160$, and $320 \mathrm{~nm}$. b Simulated reflection spectra of the structures including the top Al layer without pore and with pore $(d=65 \mathrm{~nm})$

proposed structure consisting of top $\mathrm{Al}$ layer with a pore diameter of $d=65 \mathrm{~nm}$ can strongly enhance the absorption at resonance. The observed clear redshift on the resonance wavelength can be ascribed to the result of the plasmonic effect and the changed phase shift in reflection at the top $\mathrm{Al}$ layer. To verify whether the introduced pores in the top Al layer lead to the observed absorption enhancement through the plasmonic effect, we monitor the electric field $(|E|)$ profiles at resonance in the $x-z$ plane for the two cases with and without the presence of pores on the top Al layer, as illustrated in Fig. 6c. In the structure with non-porous $\mathrm{Al}$ layer, despite the fact that a strong field enhancement can be observed within the cavity at the resonance wavelength of $559 \mathrm{~nm}$ because of the FP resonance supported by the MDM structures, part of the light is still reflected. While for the structure with porous top $\mathrm{Al}$ layer, it is observed that the electric field enhancement is not only within the cavity but also within the pore in the top $\mathrm{Al}$ layer through the plasmonic effect at the resonance wavelength of $622 \mathrm{~nm}$. As a result, light is nearly completely confined within the proposed structure, corresponding to the near-zero reflection dip shown in Fig. $6 \mathrm{~b}$.

\section{Influence of the Al Oxidation}

Notably, a $0.5-4 \mathrm{~nm}$-thick alumina layer spontaneously formed on the surface of $\mathrm{Al}$ because of air oxidation of $\mathrm{Al}$ at room temperature [36, 37]. The alumina layer serving as a stable passive layer can protect $\mathrm{Al}$ from further oxidation. Taking this situation into consideration, the reflection spectra and corresponding chromaticity coordinates of the structure with different NAA thicknesses were inspected, respectively, as shown in Fig. 7. As the thickness of the alumina layer on the surface of $\mathrm{Al}$ layers, including the top nanoporous $\mathrm{Al}$ layer and the bottom $\mathrm{Al}$ substrate, increased from $t_{0}=0$ to $4 \mathrm{~nm}$, and the reflection spectra maintained good consistency in terms of resonance wavelength and reflection efficiency. 


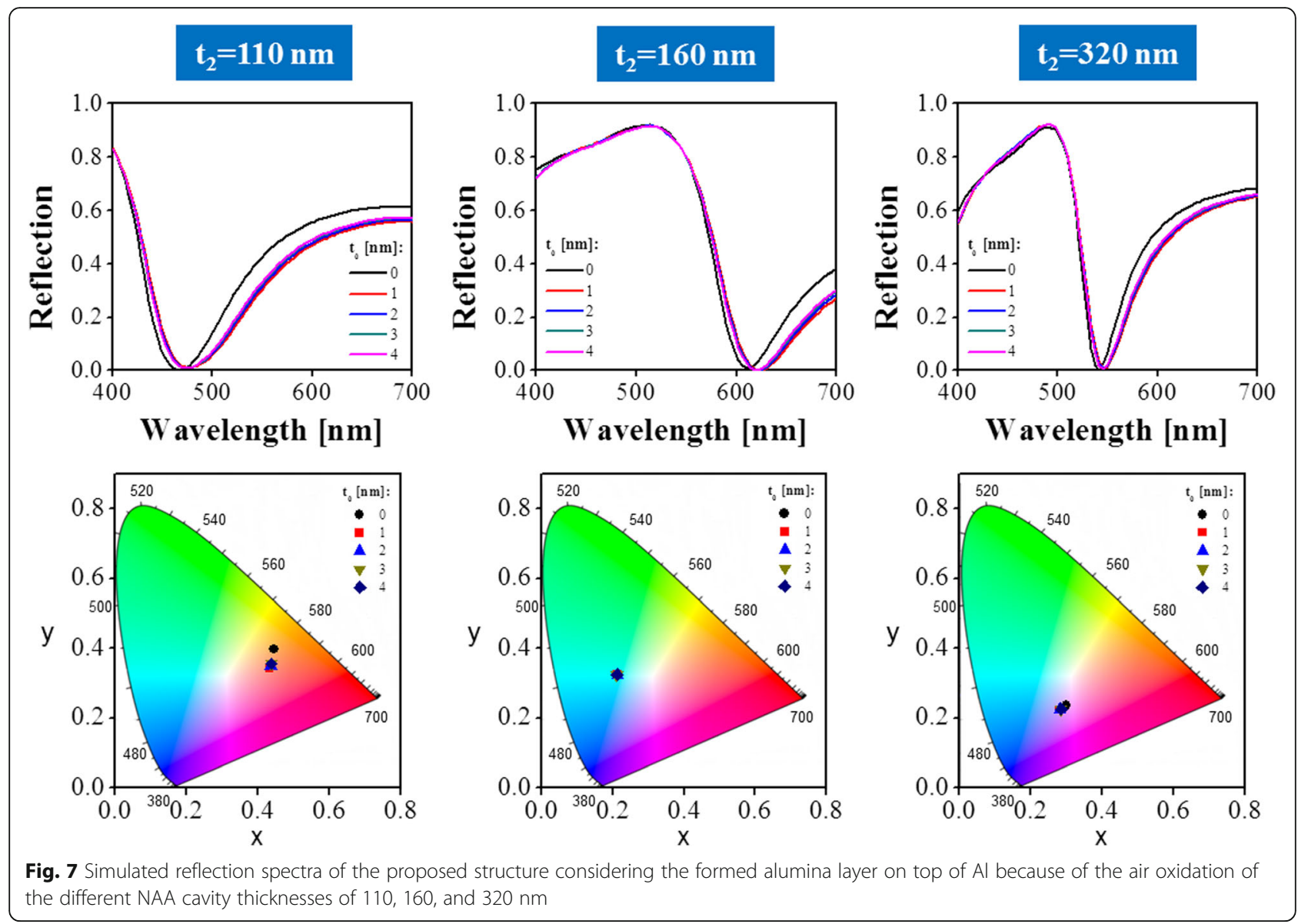

Moreover, the chromaticity coordinates indicated a stable color output after the oxidation of Al. As a result, the air oxidation of $\mathrm{Al}$ hardly affected the optical performance of the proposed structure. For comparison, the structure without an $\mathrm{Al}$ coating layer was also evaluated. As depicted in Fig. 8, the thickness of the NAA film was
$160 \mathrm{~nm}$. The gray color, which is the original color of the $\mathrm{Al}$ foil, was observed, further confirming that the proposed color filtering scheme enabled highly enhanced color purity. As indicated by the simulated and measured reflection spectra of the structure without an $\mathrm{Al}$ layer on top, no obvious resonance phenomenon was
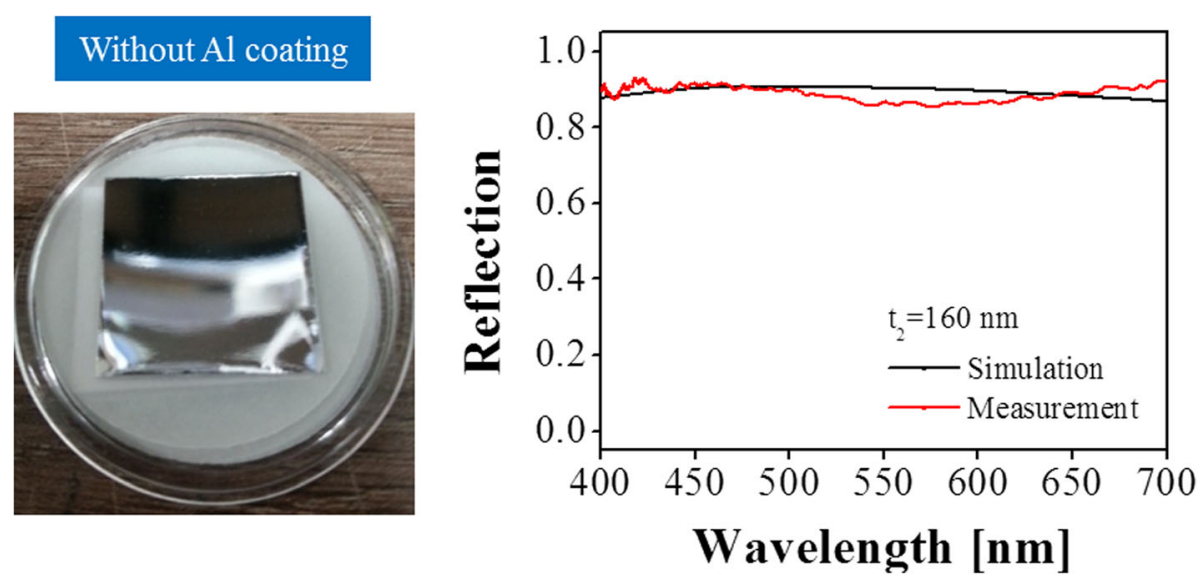

Fig. 8 Captured optical color image of the simulated and measured reflection spectra of the reference structure with no Al coating layer on top of the NAA film 
observed in the visible spectral band, resulting in the observed low-purity reflection color. Notably, the appearance of the reflection spectrum of the NAA film without $\mathrm{Al}$ coating layer was similar regardless of the thickness of the NAA film, whereas that of the Al-coated NAA film strongly depended on thickness.

\section{Conclusions}

In summary, we proposed and demonstrated an attractive method for achieving large-area color generation with high color purity through the use of a thin $\mathrm{Al}$ coating layer in conjunction with the NAA film atop an optically thick $\mathrm{Al}$ substrate. According to effective medium theory, the nanoporous layers belonging to the proposed structure, including the Al coating layer and NAA film, behave as the quasi-homogeneous media with certain effective refractive indices. As a result, the proposed structure operates as a MDM resonant structure that enables FP resonance, where the resonance wavelength corresponding to the reflection color is readily tuned by simply changing the NAA thickness. Meanwhile, by taking advantage of the top nanoporous $\mathrm{Al}$ layer, we found that the proposed structure supported the plasmonic effect, which can strongly enhance the absorption leading to the observed near-zero reflection dip. The optical performances of the proposed structure depending on its geometry were theoretically scrutinized using the FDTD-method-based tool. On the basis of optimized parameters, three samples with different NAA thicknesses were manufactured in a $2 \mathrm{~cm} \times 2 \mathrm{~cm}$ area. Through the analysis of the experimental results, the prepared samples are verified to exhibit vivid reflection colors with high reflection efficiency of up to approximately $73 \%$. The proposed approach can not only lead to a better understanding of the color-tuning mechanism of NAA-film-based configuration but also represent an important step toward the realization of cost-effective large-area color filtering devices in a large number of applications, such as display/imaging devices, photovoltaic cells, and biosensor technologies.

\section{Abbreviations}

|El: Electric field; Al: Aluminum; $\mathrm{Al}_{2} \mathrm{O}_{3}$ : Alumina; CIE: International Commission on Illumination; e-beam: Electron beam; FDTD: Finite-difference time-domain; FP: Fabry-Perot; MDM: Metal-dielectric-metal; NAA: Nanoporous anodic alumina; SEM: Scanning electron microscopy

\section{Acknowledgements}

This work was supported by the National Natural Science Foundation of China under Grant Nos. of 61604060, 61601198, Shandong Provincial Natural Science Foundation of China under Grant No. ZR2017JL027, and China Postdoctoral Science Foundation (2018M632605).

\section{Funding}

This work was funded by National Natural Science Foundation of China under Grant No. of 61604060, National Natural Science Foundation of China under Grant No. of 61601198, Shandong Provincial Natural Science Foundation of China under Grant No. ZR2017JL027, and China Postdoctoral Science Foundation under grant No. 2018M632605.

\section{Availability of Data and Materials}

The data will not be shared due to privacy and confidentiality for the purpose of patent filling.

\section{Authors' Contributions}

$Y L$ and WJY conceived and designed the experiments. YL performed the experiments. WJY did the simulation and analyzed the data. YL and WJY wrote the manuscript. ZXC and BQC contributed to the revision of resubmission. All authors read and approved the final manuscript.

\section{Competing Interests}

The authors declare that they have no competing interests.

\section{Publisher's Note}

Springer Nature remains neutral with regard to jurisdictional claims in published maps and institutional affiliations.

\section{Author details}

${ }^{1}$ School of Information Science and Engineering, University of Jinan, Jinan 250022, China. ${ }^{2}$ School of Materials Science and Engineering, University of Jinan, Jinan 250022, China.

Received: 11 November 2017 Accepted: 10 July 2018

Published online: 20 July 2018

References

1. Zhou Y, Fan H, An S, Li J, Wang J, Zhou J, Liu Y (2015) Pseudo-random arranged color filter array for controlling moiré patterns in display. Opt Express 23:29390.

2. Kitson S, Geisow A, Rudin J, Taphouse T (2011) Bright color reflective displays with interlayer reflectors. Opt Express 19:15404-15414.

3. Zhang HF, Wen L, Chen Q, Hu X, Yu Y (2015) Spatial optical crosstalk in CMOS image sensors integrated with plasmonic color filters. Opt Express 23: 21994-22003.

4. Martínez MA, Valero EM, Hernández-Andrés J, Romero J, Langfelder G (2014) Combining transverse field detectors and color filter arrays to improve multispectral imaging systems. Appl Opt 53:C14-C24.

5. Lee J-K, Kim A, Kang D-W, Lee BY (2016) Efficiency enhancement in a backside illuminated 112 um pixel CMOS image sensor via parabolic color filters. Opt Express 24:16027-16036.

6. Gupta N (2009) Biosensors technologies: acousto-optic tunable filter-based hyperspectral and polarization imagers for fluorescence and spectroscopic imaging. Methods Mol Biol 503:293-305.

7. Ellenbogen T, Seo K, Crozier KB (2012) Chromatic plasmonic polarizers for active visible color filtering and polarimetry. Nano Lett 12:1026-1031.

8. Lee K-T, Lee JY, Seo S, Guo LJ (2014) Colored ultrathin hybrid photovoltaics with high quantum efficiency. Light: Sci. Appl 3:e21e5.

9. Tan SJ, Zhang L, Zhu D, Goh XM, Wang YM, Kumar K, Qiu C-W, Yang JK (2014) Plasmonic color palettes for photorealistic printing with aluminum nanostructures. Nano Lett 14:4023-4029.

10. Medina JM, Nascimento SMC, Vukusic P (2011) Hyperspectral optical imaging of two different species of lepidoptera. Nanoscale Res Lett 6:369.

11. Kats MA, Blanchard R, Genevet P, Capasso F (2013) Nanometre optical coatings based on strong interference effects in highly absorbing media. Nature Mater 12:20-24.

12. Han JH, Kim D-Y, Kim D, Choi KC (2016) Highly conductive and flexible color filter electrode using multilayer film structure. Sci Rep 6:29341.

13. Yang C, Shen W, Zhang Y, Li K, Fang X, Zhang X, Liu X (2015) Compact multilayer film structure for angle insensitive color filtering. Sci Rep 5:9285.

14. Lee KT, Seo S, Lee JY, Guo $\sqcup$ (2014) Strong resonance effect in a Lossy medium-based optical cavity for angle robust Spectrum filters. Adv Mater 26:6324-6328.

15. Yang C, Shen W, Zhang Y, Peng H, Zhang X, Liu X (2014) Design and simulation of omnidirectional reflective color filters based on metaldielectric-metal structure. Opt Express 22:11384-11391.

16. Kanamori Y, Ozaki T, Hane K (2014) Fabrication of ultrathin color filters for three primary colors using guided-mode resonance in silicon subwavelength gratings. Opt Rev 21:723-727.

17. Xu P, Yuan X, Huang H, Yang T, Huang Y, Zhu T, Tang S, Peng W (2016) Research on design of tri-color shift device. Nanoscale Res Lett 11:485. 
18. Yokogawa S, Burgos SP, Atwater HA (2012) Plasmonic color filters for CMOS image sensor applications. Nano Lett 12:4349-4354.

19. Lee SU, Ju B-K (2017) Wide-gamut plasmonic color filters using a complementary design method. Sci Rep 7:40649.

20. Shrestha VR, Lee S-S, Kim E-S, Choi D-Y (2014) Aluminum plasmonics based highly transmissive polarization-independent subtractive color filters exploiting a nanopatch array. Nano Lett 14:6672-6678.

21. Mahani FF, Mokhtari A, Mehran M (2017) Dual mode operation, highly selective nanohole array-based plasmonic colour filters. Nanotechnology 28: 385203.

22. Chen Q, Cumming DR (2010) High transmission and low color cross-talk plasmonic color filters using triangular-lattice hole arrays in aluminum films. Opt Express 18:14056-14062.

23. Cheng F, Gao J, Luk TS, Yang X (2015) Structural color printing based on plasmonic metasurfaces of perfect light absorption. Sci Rep 5:11045.

24. Thompson GE (1997) Porous anodic alumina: fabrication, characterization and applications. Thin Solid Films 297:192-201.

25. Hotta K, Yamaguchi A, Teramae N (2012) Nanoporous waveguide sensor with optimized nanoarchitectures for highly sensitive label-free biosensing ACS Nano 6:1541-1547.

26. Zhao X, Meng G, Xu Q, Han F, Huang Q (2010) Color fine-tuning of CNTs@ AAO composite thin films via isotropically etching porous AAO before CNT growth and color modification by water infusion. Adv Mater 22:2637-2641.

27. Wang X, Akahane T, Orikasa H, Kyotani T, Fu Y (2007) Brilliant and tunable color of carbon-coated thin anodic aluminum oxide films. Appl Phys Lett 91:011908.

28. Yang C, Shen W, Zhang Y, Ye Z, Zhang X, Li K, Fang X, Liu X (2014) Color-tuning method by filling porous alumina membrane using atomic layer deposition based on metal-dielectric-metal structure. Appl Opt 53:A142-A147.

29. Li J, Zhu Z, Hu Y, Zheng J, Chu J, Huang W (2014) Numerical and experimental study of the structural color by widening the pore size of nanoporous anodic alumina. J Nanomater 2014:51.

30. Wang X, Zhang D, Zhang H, Ma Y, Jiang J (2011) Tuning color by pore depth of metal-coated porous alumina. Nanotechnology 22:305306

31. Wang X, Zhang H, Zhang D, Ma Y, Fecht HJ, Jiang J (2012) Color tuning by local sputtering metal nanolayer on microstructured porous alumina. Micros Res Tech 75:698-701.

32. Xue J, Zhou Z-K, Wei Z, Su R, Lai J, Li J, Li C, Zhang T, Wang X-H (2015) Scalable, full-colour and controllable chromotropic plasmonic printing. Nat Commun 6:8906.

33. Xiong K, Emilsson G, Maziz A, Yang X, Shao L, Jager EW, Dahlin AB (2016) Plasmonic Metasurfaces with conjugated polymers for flexible electronic paper in color. Adv Mater 28:9956-9960.

34. Bräuer R, Bryngdahl O (1994) Design of antireflection gratings with approximate and rigorous methods. Appl Opt 33:7875-7882.

35. Walls K, Chen Q, Grant J, Collins S, Cumming D, Drysdale T (2012) Narrowband multispectral filter set for visible band. Opt Express 20: 21917-21923

36. Dumas P, Dubarry-Barbe J, Riviere D, Levy Y, Corset J (1983) Growth of thin alumina film on aluminium at room temperature: a kinetic and spectroscopic study by surface plasmon excitation. Le Journal de Physique Colloques 44:C10-205-C210-208.

37. McCafferty E (2010) Introduction to corrosion science. Springer Science \& Business Media, New York.

\section{Submit your manuscript to a SpringerOpen ${ }^{\circ}$ journal and benefit from:}

- Convenient online submission

- Rigorous peer review

- Open access: articles freely available online

High visibility within the field

- Retaining the copyright to your article

Submit your next manuscript at $\boldsymbol{\nabla}$ springeropen.com 